International Journal of Applied Mathematics

Volume 31 No. 1 2018, 93-119

ISSN: 1311-1728 (printed version); ISSN: 1314-8060 (on-line version)

doi: http://dx.doi.org/10.12732/ijam.v31i1.8

\title{
DISSIPATIVE NUMERICAL METHOD FOR AN OVERHEAD CRANE MODEL WITH A FEEDBACK CONTROL FORCE IN VELOCITY
}

\author{
Siriki Ben B. Junior ${ }^{1}$, Coulibaly Adama ${ }^{2}$ \\ 1,2 University Félix Houphouët Boigny \\ Abidjan, CÔTE D'IVOIRE
}

\begin{abstract}
We present a numerical analysis on a control for the time evolution of a model of an overhead crane. This closed-loop system consists of a platform, which moves horizontally along a rail, a cable attached to the platform, and a load at its end. In the literature, it is known that it is asymptotically stable (cf. Saouri [13]). This numerical analysis concerns the dissipative finite elements method (cf. Miletic [14]) based on the $\mathbb{P}_{2}$ Lagrangian polynomials and a CrankNicholson time discretization. We prove that the numerical method dissipates the energy, analogous to the continuous case, for both discretizations semi and fully. Finally, we derive error bounds for both discretizations.
\end{abstract}

AMS Subject Classification: 35B45, 37N30, 74S10, 65N30, 35L20

Key Words: dissipative numerical method, overhead crane model, feedback control force in velocity, dissipative finite elements method, a-priori estimates, Crank-Nicholson scheme

\section{Introduction}

Cranes are essential machinery on modern world and are used to perform tasks which require the movement of heavy loads in different fields of industry such as construction, transportation or in manufacturing for the assembly of heavy components. There are several types of cranes which are selected according to the specific task to be performed. These cranes can be divided in overhead, fixed or mobile cranes.

Received: December 16, 2017

(c) 2018 Academic Publications

${ }^{\S}$ Correspondence author 
We will analyze an overhead crane model. The overhead cranes are more useful mainly inside factories to move heavy machinery or to assembly heavy equipment.

Now we describe the problem under consideration. We consider an overhead crane consisting of a motorized platform of mass $m$ moving along an horizontal bench by means of a feedback control force in velocity. A flexible cable is attached to the platform and holds a load mass $M$. The equations of motion for this system are given by:

$$
\begin{array}{lr}
y_{t t}-y_{x x}=0, & 0<x<1, t \geq 0, \\
-y_{x}(0, t)+m y_{t t}(0, t)=-\beta y_{t}(0, t), & t \geq 0, \\
y_{x}(1, t)+M y_{t t}(1, t)=0, & t \geq 0, \\
y(x, 0)=y_{0}(x), y_{t}(x, 0)=y_{1}(x), & 0<x<1,
\end{array}
$$

where $\beta$ is a non negative constant; $y(x, t)$ represents the transversal displacement of the cable whose curvilinear abscissa is $x$ at time $t$.

Well-posedness of this system and asymptotical stability of the system were established in Saouri [13], using semigroup theory on an equivalent first order system (in time), a carefully designed Lyapunov functional, and LaSalle's invariance principle.

The goal of this paper is to develop and analyze a dissipative finite element method (cf. Miletic [14]) for the control system. Our main focus will be on preserving the correct large-time behavior (i.e. dissipativity) in the numerical scheme.

The rest of the paper is organized as follows. In Section 2, the well-posedness of the system (1)-(4) is established. Section 3 is devoted to the numerical resolution of (1)-(4) by the method of dissipative finite elements (cf. Miletic [14]). Finally, in Section 4, a-priori estimates of errors of semi discrete and fully discrete approximations are given.

\section{Well-posedness of the system}

Let us define the Hilbert space

$$
\mathcal{H}=\left\{U=(y, z, u, \nu)^{\top}: y \in H^{1}(0,1), z \in L^{2}(0,1), u, \nu \in \mathbb{R}\right\},
$$

with the inner product

$$
\langle U, \tilde{U}\rangle_{\mathcal{H}}=\int_{0}^{1}\left(y_{x} \tilde{y}_{x}+z \tilde{z}\right) d x+M u \tilde{u}+m \nu \tilde{\nu}+\rho\left(\int_{0}^{1} z d x+M u\right.
$$




$$
+m \nu+\beta y(0, t))\left(\int_{0}^{1} \tilde{z} d x+M \tilde{u}+m \tilde{\nu}+\beta \tilde{y}(0, t)\right)
$$

where $U=(y, z, u, \nu)^{\top}, \tilde{U}=(\tilde{y}, \tilde{z}, \tilde{u}, \tilde{\nu})^{\top} \in \mathcal{H}, \rho>0$ and $\|U\|_{\mathcal{H}}$ denotes the corresponding norm.

Let the linear operator $A$ with the domain

$$
\begin{aligned}
D(A)=\{ & (y, z, u, \nu)^{\top} \in \mathcal{H}: y \in H^{2}(0,1), z \in H^{1}(0,1), u=z(1), \\
& \nu=z(0)\}
\end{aligned}
$$

be given by:

$$
A\left(\begin{array}{c}
y \\
z \\
u \\
\nu
\end{array}\right)=\left(\begin{array}{c}
z \\
y_{x x} \\
-\frac{1}{M} y_{x}(1) \\
\frac{1}{m}\left(y_{x}(0)-\beta \nu\right) .
\end{array}\right) .
$$

With the previous notations, the equations (1)-(4) can be formally written as the form:

$$
\dot{U}=A U, \quad U(0) \in \mathcal{H} .
$$

We have the following theorem:

Theorem 1. (Saouri [13]) The operator $A$, defined by (6)-(7), is $m$ dissipative. Then $A$ generates a $\mathcal{C}_{0}$-semigroup of contractions on $\mathcal{H}$.

Proof. For the proof, see Saouri [13], Chapter 4.

Remark 2. (Saouri [13]) It follows from the previous theorem that the problem (8) has a unique strong solution $U \in C\left(\mathbb{R}_{+}, D(A)\right) \cap C^{1}\left(\mathbb{R}_{+}, \mathcal{H}\right)$, if $U_{0} \in D(A)$ and a unique weak solution $U \in C\left(\mathbb{R}_{+}, \mathcal{H}\right)$ if $U_{0} \in \mathcal{H}$.

Finally, we conclude that the problem defined by the system (1)-(4) is well posed. 


\section{Numerical resolution}

\subsection{Weak formulation}

Consider $H_{E}^{1}(0,1):=H^{1}(0,1) \cap W$ and $L_{E}^{2}(0,1):=L^{2}(0,1) \cap W$, where $W:=$ $\{w: w(1)=0\}$. In order to determine the weak formulation of (1)-(4), the following initial conditions are assumed:

$$
\begin{gathered}
y(0)=y_{0} \in H_{E}^{1}(0,1), \\
y_{t}(0)=y_{1} \in L_{E}^{2}(0,1) .
\end{gathered}
$$

Let $t \in\left[0,+\infty\left[, w \in H_{E}^{1}(0,1)\right.\right.$ and $x \in[0,1]$. Then we have:

$$
(1) \Longrightarrow \int_{0}^{1}\left(y_{t t}(x, t) w(x)-y_{x x}(x, t) w(x)\right) d x=0 \text {. }
$$

Performing partial integration and using the expressions (2) and (3), we obtain:

$$
\begin{aligned}
(1) \Longrightarrow & \int_{0}^{1}\left(y_{t t}(x, t) w(x)+y_{x}(x, t) w_{x}(x)\right) d x+m y_{t t}(0, t) w(0) \\
& +\beta y_{t}(0, t) w(0)=0 .
\end{aligned}
$$

Let $H$ be a Hilbert space with the inner product defined by:

$$
\begin{aligned}
H & :=\mathbb{R} \times \mathbb{R} \times L_{E}^{2}(0,1), \\
\langle\hat{\omega}, \hat{\phi}\rangle_{H} & :=\int_{0}^{1} \omega_{3} \phi_{3} d x+m \omega_{1} \phi_{1},
\end{aligned}
$$

where $\hat{\omega}=\left(\omega_{1}, \omega_{2}, \omega_{3}\right), \hat{\phi} \in H$. Next, we consider another Hilbert space $V$ and its inner product defined as follows:

$$
\begin{aligned}
V & :=\left\{\hat{\omega}=\left(\omega(0), \omega_{x}(0), \omega\right): \omega \in H_{E}^{1}(0,1)\right\}, \\
\langle\hat{\omega}, \hat{\phi}\rangle_{V} & :=\left\langle\left(\omega_{3}\right)_{x},\left(\phi_{3}\right)_{x}\right\rangle_{L^{2}(0,1)} .
\end{aligned}
$$

$V$ is densely embedded in $H$. Therefore taking $H$ as a pivot space, we obtain a Gelfand triple $V \subset H \subset V^{\prime}$. Moreover, consider the following bilinear forms:

$$
\begin{array}{rlrl}
a: V \times V & \rightarrow \mathbb{R} \quad b: H \times H & \rightarrow \mathbb{R} \\
(\hat{\omega}, \hat{\phi}) & \mapsto\langle\hat{\omega}, \hat{\phi}\rangle_{V} \quad(\hat{\omega}, \hat{\phi}) \mapsto \beta \omega_{1} \phi_{1} .
\end{array}
$$

We have the following definition: 
Definition 3. Let $T>0$ be fixed. Function $\hat{y}=\left(y(0), y_{x}(0), y\right)$ is said to be the weak solution of (1)-(4) on $[0, T]$ if

$$
\hat{y} \in L^{2}(0, T ; V) \cap H^{1}(0, T ; H) \cap H^{2}\left(0, T ; V^{\prime}\right)
$$

and satisfies for almost every $t \in(0, T)$ :

$$
V^{\prime}\left\langle\hat{y}_{t t}, \hat{w}\right\rangle_{V}+a(\hat{y}, \hat{w})+b\left(\hat{y}_{t}, \hat{w}\right)=0, \forall t \in(0, T), \hat{w} \in V,
$$

with the initial conditions:

$$
\begin{aligned}
& \hat{y}(0)=\hat{y}_{0}=\left(y_{0}(0),\left(y_{0}\right)_{x}(0), y_{0}\right) \in V, \\
& \hat{y}_{t}(0)=\hat{z}_{0}=\left(y_{1}(0),\left(y_{1}\right)_{x}(0), y_{1}\right) \in H .
\end{aligned}
$$

\subsubsection{Existence and Uniqueness results}

In this paragraph, we use the intermediate spaces $[X, Y]_{\theta}$. For the definition of these spaces, see Section 2.1 of Lions et al. [8].

Lemma 4. Let $X$ and $Y$ be Hilbert spaces, such that $X$ is dense and continuously embedded in $Y$. Suppose that:

$$
\begin{gathered}
y \in L^{2}(0, T ; X), \\
y_{t} \in L^{2}(0, T ; Y) .
\end{gathered}
$$

Then we have

$$
y \in C\left([0, T] ;[X, Y]_{\frac{1}{2}}\right)
$$

after, possibly, a modification on a set of measure zero.

Additionally, the following 'Duality Theorem' (cf. Lions et al. [8], Chapter 6, pp. 29) will be needed in the proof of Theorem 7.

Lemma 5. Let $X$ and $Y$ be Hilbert spaces, such that $X$ is dense and continuously embedded in $Y$. For all $\theta \in(0,1)$, we have

$$
[X, Y]_{\theta}^{\prime}=\left[Y^{\prime}, X^{\prime}\right]_{1-\theta} .
$$

Lemma 6. Let

$$
H_{E}^{1}(0,1):=\left\{y \in H^{1}(0,1): y(1)=0\right\} .
$$

Then, there exists a set of functions $\left\{w_{k}\right\}_{k=1}^{\infty}$ that is an orthogonal basis of $H_{E}^{1}(0,1)$ and an orthonormal basis of $L_{E}^{2}(0,1)$. 
Proof. Let $L$ be a second order differential operator given by:

$$
L y=y_{x x}
$$

Consider the following problem:

$$
\begin{aligned}
& L y(x)=-f(x), x \in(0,1), \\
& y(1)=y_{x}(0)=0 .
\end{aligned}
$$

Assuming that $f \in L_{E}^{2}(0,1)$, we recall that a weak solution of this problem is defined to be $y \in H_{E}^{1}(0,1)$ such that

$$
\int_{0}^{1} y_{x} w_{x} d x=\int_{0}^{1} f w d x
$$

for all $w \in H_{E}^{1}(0,1)$.

The bilinear symmetric form

$$
a_{1}(\phi, w)=\int_{0}^{1} \phi_{x} w_{x} d x
$$

is coercive and bounded on $H_{E}^{1}(0,1)$. From the Lax-Milgram theorem, it follows that weak formulation (i) has a unique solution $y \in H_{E}^{1}(0,1)$. Then, we have

$$
y=-L^{-1} f .
$$

Operator $L^{-1}: L_{E}^{2}(0,1) \rightarrow H_{E}^{1}(0,1)$ is linear and bounded. Moreover, let $T:=-\mathcal{I} L^{-1} \in \mathcal{L}\left(L_{E}^{2}(0,1)\right)$, where $\mathcal{I}$ is the embedding of $H_{E}^{1}(0,1)$ in $L_{E}^{2}(0,1)$ which is compact. $T$ is compact because product of two operators whose one is compact.

Now, we show that $T$ is symmetric. Let us consider $f, g \in L_{E}^{2}(0,1)$ and set $w=-L^{-1} g$ in (i).

We obtain:

$$
\begin{aligned}
& \langle f, T g\rangle_{L^{2}(0,1)}=\left\langle f,-L^{-1} g\right\rangle_{L^{2}(0,1)}=a_{1}\left(L^{-1} f, L^{-1} g\right) \\
& =a_{1}\left(L^{-1} g, L^{-1} f\right)=\left\langle g,-L^{-1} f\right\rangle_{L^{2}(0,1)}=\langle g, T f\rangle_{L^{2}(0,1)}
\end{aligned}
$$

Then $T$ is symmetric. Furthermore, $T$ is positive definite because $a_{1}$ is coercive. Then, there exists a countable orthonormal basis $\left\{w_{k}\right\}_{k=1}^{\infty}$ of $L_{E}^{2}(0,1)$ consisting of eigenvectors of $T$. Additionally, these eigenvectors are in $H_{E}^{1}(0,1)$ according to the definition of $T$. From the weak formulation, one can see that the basis $\left\{w_{k}\right\}_{k=1}^{\infty}$ is orthogonal in $H_{E}^{1}(0,1)$ with respect to the inner product $a_{1}(.,$.$) .$ 


\section{Theorem 7.}

(a) The weak formulation (14)-(16) has a unique solution $\hat{y}$.

(b) The additional regularity holds for the weak solution $\hat{y}$ :

$$
\begin{array}{r}
\hat{y} \in L^{\infty}(0, T ; V), \hat{y}_{t} \in L^{\infty}(0, T ; H), \\
\hat{y} \in C\left([0, T] ;[V, H]_{\frac{1}{2}}\right), \\
\hat{y}_{t} \in C\left([0, T] ;[V, H]_{\frac{1}{2}}^{\prime}\right) .
\end{array}
$$

Proof.

\section{Step 1: Existence of the solution of the weak problem.}

Let $\left(\hat{w}_{k}\right)_{k}$ be a sequence of functions that is an orthonormal basis for $H$ and an orthogonal basis for $V$. Such basis exists and its construction is given by Lemma 6. We introduce the following finite dimensional spaces:

$$
\hat{W}_{n}:=\left\langle\hat{w}_{1}, \ldots, \hat{w}_{n}\right\rangle, \forall n \in \mathbb{N} .
$$

Let $n \in \mathbb{N}$ and the Galerkin approximation $\hat{y}_{n}(t) \in \hat{W}_{n}$ :

$$
\hat{y}_{n}(t)=\left(y_{n}(0),\left(y_{n}\right)_{x}(0), y_{n}\right)=\sum_{k=1}^{n} d_{n}^{k}(t) \hat{w}_{k}
$$

with $d_{n}^{k}(t) \in \mathbb{R}$, which solves (11) on $\hat{W}_{n}$ :

$$
\left\langle\left(\hat{y}_{n}\right)_{t t}, \hat{w}\right\rangle_{H}+a\left(\hat{y}_{n}, \hat{w}\right)+b\left(\left(\hat{y}_{n}\right)_{t}, \hat{w}\right)=0
$$

with the initial conditions:

$$
\begin{aligned}
& \hat{y}_{n}(0)=\hat{y}_{0 n}=\sum_{k=1}^{n}\left\langle\hat{y}_{0}, \hat{w}_{k}\right\rangle_{V} \hat{w}_{k}, \quad \hat{y}_{0 n} \underset{n \rightarrow \infty}{\longrightarrow} \hat{y}_{0} \text { in } V, \\
& \left(\hat{y}_{n}\right)_{t}(0)=\hat{z}_{0 n}=\sum_{k=1}^{n}\left\langle\hat{z}_{0}, \hat{w}_{k}\right\rangle_{H} \hat{w}_{k}, \quad \hat{z}_{0 n} \underset{n \rightarrow \infty}{\longrightarrow} \hat{z}_{0} \text { in } H .
\end{aligned}
$$

Thus we obtain a linear system of second order differential equations. After rewriting it as a system of first order differential equations, the Cauchy-Lipschitz theorem implies that this system has a unique solution $\hat{y}_{n} \in C^{2}([0, T] ; V)$.

Next, let us define an energy functional for the trajectory $\hat{y}$ :

$$
\hat{E}(t ; \hat{y}):=\frac{1}{2}\left[\|\hat{y}\|_{V}^{2}+\left\|\hat{y}_{t}\right\|_{H}^{2}\right] .
$$


Taking $\hat{w}=\left(\hat{y}_{n}\right)_{t}$ in $(22)$ and using the smoothness of $\hat{y}_{n}$, we obtain:

$$
\frac{d}{d t} \hat{E}\left(t ; \hat{y}_{n}\right)=-\beta\left(\hat{y}_{n, 1}\right)_{t}^{2} \leq 0 .
$$

Consequently,

$$
\hat{E}\left(t ; \hat{y}_{n}\right) \leq \hat{E}\left(t ; \hat{y}_{0 n}\right), \quad t \geq 0,
$$

and since the sequences $\left(\hat{y}_{0 n}\right)_{n}$ and $\left(\hat{z}_{0 n}\right)_{n}$ are convergent, then:

$$
\begin{aligned}
& \hat{y}_{n} \text { is bounded in } C([0, T] ; V), \\
& \left(\hat{y}_{n}\right)_{t} \text { is bounded in } C([0, T] ; H) .
\end{aligned}
$$

Using these boundedness results, it holds:

$$
\forall \hat{w} \in V,\left|a\left(\hat{y}_{n}(t), \hat{w}\right)+b\left(\left(\hat{y}_{n}\right)_{t}(t), \hat{w}\right)\right| \leq D_{1}\|\hat{w}\|_{V},
$$

almost everywhere on $(0, T)$, with some constant $D_{1}>0$ independent of $n$.

Let $n \in \mathbb{N}$ and $\hat{w} \in V$ such that $\hat{w}=\hat{\phi}_{1}+\hat{\phi}_{2}$, where $\hat{\phi}_{1} \in \hat{W}_{n}$ and $\hat{\phi}_{2} \in \hat{W}_{n}^{\top}$, orthogonal of $\hat{W}_{n}$ in $H$. Then we have:

$$
\begin{aligned}
\left\langle\left(\hat{y}_{n}\right)_{t t}, \hat{w}\right\rangle_{H} & =\left\langle\left(\hat{y}_{n}\right)_{t t}, \hat{\phi}_{1}\right\rangle_{H} \\
& =-a\left(\hat{y}_{n}, \hat{\phi}_{1}\right)-b\left(\left(\hat{y}_{n}\right)_{t}, \hat{\phi}_{1}\right) \\
& \leq D_{1}\left\|\hat{\phi}_{1}\right\|_{V} \leq D_{1}\|\hat{w}\|_{V} .
\end{aligned}
$$

This shows that the function $\left(\hat{y}_{n}\right)_{t t}$ is bounded in $C\left([0, T] ; V^{\prime}\right)$. Due to the Eberlein-Smuljan theorem, there exist a subsequence $\left(\hat{y}_{n_{l}}\right)_{l}$, and functions $\hat{y} \in$ $L^{2}(0, T ; V), \hat{y}_{t} \in L^{2}(0, T ; H), \hat{y}_{t t} \in L^{2}\left(0, T ; V^{\prime}\right)$ such that:

$$
\begin{array}{r}
\left(\hat{y}_{n_{l}}\right)_{l} \rightarrow \hat{y} \text { in } L^{2}(0, T ; V), \\
\left(\left(\hat{y}_{n_{l}}\right)_{t}\right)_{l} \rightarrow \hat{y}_{t} \text { in } L^{2}(0, T ; H), \\
\left(\left(\hat{y}_{n_{l}}\right)_{t t}\right)_{l} \rightarrow \hat{y}_{t t} \text { in } L^{2}\left(0, T ; V^{\prime}\right) .
\end{array}
$$

Moreover, (31) yields

$$
\left(\left(\hat{y}_{n_{l}, i}\right)_{t}\right)_{l} \longrightarrow\left(\hat{y}_{n, i}\right)_{t}
$$

for $i=1,2$ and for almost every $t$ in $[0, T]$. 
Let $n_{0} \in \mathbb{N}$. Consider the function $\hat{\varphi} \in L^{2}\left(0, T, \hat{W}_{n_{0}}\right)$ such that:

$$
\hat{\varphi}(t, x)=\sum_{j=1}^{n_{0}} \alpha_{j}(t) w_{j}(x),
$$

where $\alpha_{j} \in L^{2}(0, T, \mathbb{R})$, and for all $n_{l} \geq n_{0}$, equation (22) yields:

$$
\int_{0}^{T}\left(\left\langle\left(\hat{y}_{n_{l}}\right)_{t t}, \hat{\varphi}\right\rangle_{H}+a\left(\hat{y}_{n_{l}}, \hat{\varphi}\right)+b\left(\left(\hat{y}_{n_{l}}\right)_{t}, \hat{\varphi}\right)\right) d t=0 .
$$

Passing to the limit in (34), and using (31), one obtains:

$$
\int_{0}^{T} V^{\prime}\left\langle\hat{y}_{t t}, \hat{\varphi}\right\rangle_{V}+a(\hat{y}, \hat{\varphi})+b\left(\hat{y}_{t}, \hat{\varphi}\right) d t=0
$$

However, functions of the form (33) are dense in $L^{2}(0, T ; V)$ and hence (35) holds for all $\hat{\varphi}$ in $L^{2}(0, T ; V)$. This implies that (14) is satisfied almost everywhere on $[0, T]$. Therefore $\hat{y}$ solves the weak formulation.

\section{Step 2: Regularity.}

From the construction of the weak solution and (28), $\hat{y}$ satisfies (18). Using Lemma 4, we obtain (19), after, possibly, a modification on a set of measure zero. Moreover, regularity (20) follows from Lemmas 4 and 5.

\section{Step 3: Verification of initial conditions.}

We show that $\hat{y}$ satisfies initial conditions. For this purpose, equation (14) is integrated by parts (in time), with $\hat{w} \in C^{2}([0, T] ; V)$ such that $\hat{w}(T)=0$ and $\hat{w}_{t}(T)=0$ :

$$
\begin{aligned}
\int_{0}^{T} & {\left[\left\langle\hat{y}, \hat{w}_{t t}\right\rangle_{H}+a(\hat{y}, \hat{w})+b\left(\hat{y}_{t}, \hat{w}\right)\right] d \tau=\int_{0}^{T}\left[\left\langle\hat{y}, \hat{w}_{t t}\right\rangle_{H}\right.} \\
& \left.-V_{V^{\prime}}\left\langle\hat{y}_{t t}, \hat{w}\right\rangle_{V}\right] d \tau=-\left\langle\hat{y}(0), \hat{w}_{t}(0)\right\rangle_{H}+V^{\prime}\left\langle\hat{y}_{t}(0), \hat{w}(0)\right\rangle_{V} .
\end{aligned}
$$

Similarly, for a fixed $n$, it follows from (22):

$$
\begin{gathered}
\int_{0}^{T}\left[\left\langle\hat{y}_{n}, \hat{w}_{t t}\right\rangle_{H}+a\left(\hat{y}_{n}, \hat{w}\right)+b\left(\left(\hat{y}_{t}\right)_{n}, \hat{w}\right)\right] d \tau \\
=-\left\langle\hat{y}_{0 n}, \hat{w}_{t}(0)\right\rangle_{H}+\left\langle\hat{z}_{0 n}, \hat{w}(0)\right\rangle_{H} .
\end{gathered}
$$


Using (23)-(24) and (31), passing to the limit in (37) along the convergent subsequence $\left(y_{n_{l}}\right)_{l}$, one obtains:

$$
\int_{0}^{T}\left[\left\langle\hat{y}, \hat{w}_{t t}\right\rangle_{H}+a(\hat{y}, \hat{w})+b\left(\hat{y}_{t}, \hat{w}\right)\right] d \tau=-\left\langle\hat{y}_{0}, \hat{w}_{t}(0)\right\rangle_{H}+\left\langle\hat{z}_{0}, \hat{w}(0)\right\rangle_{H} .
$$

Comparing (36) and (38), we obtain by identification that $\hat{y}(0)=\hat{y}_{0}, \hat{y}_{t}(0)=\hat{z}_{0}$.

\section{Step 4: Uniqueness of the solution.}

Consider $\hat{y}$ solving (14) with zero initial conditions. Let $s \in(0, T)$ be fixed, and set

$$
\hat{Y}(t):=\left\{\begin{array}{l}
-\int_{t}^{s} \hat{y}(\tau) d \tau, \quad t<s \\
0 \quad \text { else. }
\end{array}\right.
$$

We have:

$$
\int_{0}^{s}\left({ }^{\prime}\left\langle\hat{y}_{t t}(\tau), \hat{Y}(\tau)\right\rangle_{V}+a(\hat{y}(\tau), \hat{Y}(\tau))+b\left(\hat{y}_{t}(\tau), \hat{Y}(\tau)\right)\right) d \tau=0 .
$$

Performing partial integrations, we obtain:

$$
-\frac{1}{2} \int_{0}^{s} \frac{d}{d t}\|y(\tau)\|_{H}^{2}+\frac{1}{2} \int_{0}^{s} \frac{d}{d t} a(\hat{Y}(\tau), \hat{Y}(\tau)) d \tau-\int_{0}^{s} b(\hat{y}(\tau), \hat{y}(\tau)) d \tau=0 .
$$

From (40) follows:

$$
\frac{1}{2} \int_{0}^{s} \frac{d}{d t}\|\hat{y}(\tau)\|_{H}^{2}-\frac{1}{2} \int_{0}^{s} \frac{d}{d t} a(\hat{Y}(\tau), \hat{Y}(\tau)) d \tau=-\int_{0}^{s} b(\hat{y}(\tau), \hat{y}(\tau)) d \tau \leq 0 .
$$

Then we have:

$$
\|\hat{y}(s)\|_{H}^{2}+a(\hat{Y}(0), \hat{Y}(0)) \leq 0
$$

Hence $\hat{y}(s)=0$ and $\hat{Y}(0)=0$ ( $a$ is coercive). Since $s \in(0, T)$ is arbitrary, then $\hat{y} \equiv 0$.

\subsubsection{High regularity results}

In this subsection, we demonstrate that even stronger continuity holds for the weak solution $\hat{y}$ solving (14)-(16). 
Theorem 8. After, possibly a modification on a set of measure zero, the weak solution $\hat{y}$ of (14)-(16) satisfies

$$
\begin{gathered}
\hat{y} \in C([0, T] ; V), \\
\hat{y}_{t} \in C([0, T] ; H) .
\end{gathered}
$$

A definition and a lemma are stated before demonstrating this theorem.

Definition 9. Let $Y$ be a Banach space. Then

$$
\begin{aligned}
& C_{w}([0, T] ; Y):=\left\{w \in L^{\infty}(0, T ; Y):\right. \\
& \left.\quad t \mapsto\langle f, w(t)\rangle \text { is continuous on }[0, T], \forall f \in Y^{\prime}\right\}
\end{aligned}
$$

denotes the space of weakly continuous functions with values in $Y$.

The following lemma was stated and proved in Lions et al. [8] (Chapter 8, pp. 275).

Lemma 10. Let $X, Y$ be Banach spaces, $X \subset Y$ with continuous injection, $X$ reflexive. Then we have:

$$
L^{\infty}(0, T ; X) \cap C_{w}(0, T ; Y)=C_{w}(0, T ; X) .
$$

\section{Proof. Proof of Theorem 8.}

This proof is an adaptation of standard strategies to the situation at hand (see Section 8.4 in Lions et al. [8] and Section 2.4 in Temam [11]). Using Lemma 10 with $X=V$ and $Y=H$, it follows from (18) and (19) that $\hat{y} \in C_{w}([0, T] ; V)$. Similarly, (18) and (20) imply $\hat{y}_{t} \in C_{w}([0, T] ; H)$.

Now, consider the scalar cut-off function $\xi \in C^{\infty}(\mathbb{R})$ such that it equals 1 on some interval $J \subset \subset[0, T]$, and 0 on $\mathbb{R} \backslash[0, T]$. Then the function $\xi \hat{y}: \mathbb{R} \rightarrow V$ is compactly supported. Let $\eta^{\varepsilon}: \mathbb{R} \rightarrow \mathbb{R}$ be a standard mollifier in time. For example, $\eta^{\varepsilon}$ may be given by:

$$
\eta^{\varepsilon}:=\frac{1}{\varepsilon} \eta\left(\frac{t}{\varepsilon}\right),
$$

where

$$
\eta(t):= \begin{cases}e^{-\frac{1}{1-|t|^{2}}}, & |t|<1 \\ 0, & |t| \geq 1\end{cases}
$$


The following definition is introduced:

$$
\hat{y}^{\varepsilon}:=\eta^{\varepsilon} * \xi \hat{y} \in C_{c}^{\infty}(\mathbb{R}, V),
$$

$\hat{y}^{\varepsilon}$ converges to $\hat{y}$ in $V$, and $\hat{y}_{t}^{\varepsilon}$ to $\hat{y}_{t}$ in $H$ almost everywhere on $J$. Then $\hat{E}\left(t ; \hat{y}^{\varepsilon}\right)$ converges to $\hat{E}(t ; \hat{y})$ almost everywhere on $J$. Since $\hat{y}^{\varepsilon}$ is smooth, one has:

$$
\frac{d}{d t} \hat{E}\left(t ; \hat{y}^{\varepsilon}\right)=-\beta\left(\hat{y}_{t}^{\varepsilon}\right)_{1}^{2} \leq 0
$$

Passing to the limit, when $\varepsilon \rightarrow 0$,

$$
\frac{d}{d t} \hat{E}(t ; \hat{y})=-\beta\left(\hat{y}_{t}\right)_{1}^{2} \leq 0
$$

holds in the sense of distributions on $J$. Since $J$ is arbitrary, (46) holds on all compact subintervals of $(0, T)$.

For a fixed $t$, let $\lim _{n \rightarrow \infty} t_{n}=t$ and the sequence $\left(\pi_{n}\right)_{n}$ be defined by:

$$
\pi_{n}:=\frac{1}{2}\left[\left\|\hat{y}-\hat{y}\left(t_{n}\right)\right\|_{V}^{2}+\left\|\hat{y}_{t}-\hat{y}_{t}\left(t_{n}\right)\right\|_{H}^{2}\right] .
$$

Then we have:

$$
\pi_{n}:=\hat{E}(t ; \hat{y})+\hat{E}\left(t_{n} ; \hat{y}\right)-\left\langle\hat{y}(t), \hat{y}\left(t_{n}\right)\right\rangle_{V}-\left\langle\hat{y}_{t}(t), \hat{y}_{t}\left(t_{n}\right)\right\rangle_{H} .
$$

Due to the $t$-continuity of the energy function $\hat{E}$, and using weak continuity of $\hat{y}$ and $\hat{y}_{t}$, we obtain:

$$
\lim _{n \rightarrow \infty} \pi_{n}=0
$$

Finally, this implies that:

$$
\begin{aligned}
\lim _{n \rightarrow \infty}\left\|\hat{y}(t)-\hat{y}\left(t_{n}\right)\right\|_{V} & =0, \\
\lim _{n \rightarrow \infty}\left\|\hat{y}_{t}(t)-\hat{y}_{t}\left(t_{n}\right)\right\|_{H} & =0 .
\end{aligned}
$$

which proves the theorem.

\subsection{Dissipative FEM method}

The goal of this section is to develop a stable and convergent numerical method which faithfully describes the behavior of the system. We know, in fact, that the energy of the system decreases in time:

$$
\frac{d}{d t} \hat{E}(t ; y):=-\beta y_{t}(0)^{2} \leq 0 .
$$


Therefore, it is important that the corresponding numerical method preserves the structural property of dissipativity: for longtime computations, the numerical scheme must be convergent in classical sense, but also yield the correct large time limit. Moreover, the dissipativity of the scheme implies immediately unconditional stability.

\subsubsection{Semi-discrete scheme: space discretization}

Let $V_{h} \subset H_{E}^{1}(0,1)$ be an arbitrary chosen finite dimensional space. We obtain the following approximating problem:

Problem $G^{h}:$ Find $y_{h} \in C^{2}\left([0,+\infty), V_{h}\right)$, i.e. $\hat{y}_{h}=\left(y_{h}(0),\left(y_{h}\right)_{x}(0), y_{h}\right) \in C^{2}([0,+\infty), V)$ verifying:

$$
\begin{aligned}
& \int_{0}^{1}\left(\left(y_{h}\right)_{t t}(t)(x) w_{h}(x)+\left(\left(y_{h}\right)(t)\right)_{x}(x)\left(w_{h}\right)_{x}(x)\right) d x \\
& +m\left(y_{h}\right)_{t t}(t)(0) w_{h}(0)+\beta\left(y_{h}\right)_{t}(t)(0) w_{h}(0)=0,
\end{aligned}
$$

with the following initial conditions:

$$
\begin{aligned}
y_{h}(., 0) & =y_{h}^{0} \in V_{h}, \\
\left(y_{h}\right)_{t}(., 0) & =y_{h}^{1} \in V_{h} .
\end{aligned}
$$

Discretize $[0,1]$ in $p$ subintervals of same length. $V_{h} \subset H^{1}(0,1)$ then its elements are globally $C[0,1]$. Let us consider:

$$
V_{h}:=\left\{\phi \in C[0 ; 1]: \phi_{\mid\left[x_{k} ; x_{k+1}\right]} \in \mathbb{P}_{2}(\mathbb{R}), k=0, \ldots, p-1\right\},
$$

with $x_{k}=k h, k=0,1, \ldots, p$. Then $\operatorname{dim} V_{h}=2 p$ and note

$$
V_{h}=\left\langle\phi_{1}, \phi_{2}, \ldots, \phi_{2 p}\right\rangle,
$$

where $\phi_{i}, i=1, \ldots, 2 p$, are the associated basis functions at nodes $x_{j}, j=$ $0, \frac{1}{2}, 1, \frac{3}{2}, 2, \ldots, p-\frac{1}{2}$, respectively.

In this basis, $y_{h}(x, t)=y_{h}(t)(x)=\sum_{j=1}^{2 p} Y_{j}(t) \phi_{j}(x)$. Replacing $y_{h}$ by his expression in (51), one obtains:

$$
\begin{array}{r}
\sum_{j=1}^{2 p}\left[\left(\int_{0}^{1} \phi_{i} \phi_{j} d x+m \phi_{i}(0) \phi_{j}(0)\right)\left(Y_{j}\right)_{t t}(t)+\int_{0}^{1}\left(\phi_{i}\right)_{x}\left(\phi_{j}\right)_{x} d x Y_{j}(t)\right. \\
\left.+\beta \phi_{i}(0) \phi_{j}(0)\left(Y_{j}\right)_{t}(t)\right]=0
\end{array}
$$


for all $i=1,2, \ldots, 2 p$. Finally, we obtain an ordinary differential equation:

$$
\mathbb{A} Y_{t t}(t)+\beta \mathbb{B} Y_{t}(t)+\mathbb{K} Y(t)=0
$$

where

$$
\mathbb{A}=\left(a_{i j}\right)_{1 \leq i, j \leq 2 p}, \mathbb{B}=\left(b_{i j}\right)_{1 \leq i, j \leq 2 p}, \mathbb{K}=\left(k_{i j}\right)_{1 \leq i, j \leq 2 p}, Y=\left(Y_{i}\right)_{1 \leq i \leq 2 p}
$$

and

$$
a_{i j}=\int_{0}^{1} \phi_{i} \phi_{j} d x+m \phi_{i}(0) \phi_{j}(0), b_{i j}=\phi_{i}(0) \phi_{j}(0), k_{i j}=\int_{0}^{1}\left(\phi_{i}\right)_{x}\left(\phi_{j}\right)_{x} d x .
$$

Derivation of element matrices. The element matrices are:

$$
\begin{gathered}
\mathbb{A}_{e}=\frac{h}{2}\left(\begin{array}{ccc}
\frac{4}{15} & \frac{2}{15} & -\frac{1}{15} \\
\frac{2}{15} & \frac{16}{15} & \frac{2}{15} \\
-\frac{1}{15} & \frac{2}{15} & \frac{4}{15}
\end{array}\right), \mathbb{K}_{e}=\frac{2}{h}\left(\begin{array}{ccc}
\frac{7}{6} & -\frac{4}{3} & \frac{1}{6} \\
-\frac{4}{3} & \frac{8}{3} & -\frac{4}{3} \\
\frac{1}{6} & -\frac{4}{3} & \frac{7}{6}
\end{array}\right), \\
\mathbb{B}_{e}=\mathbb{O}_{3} .
\end{gathered}
$$

Remark 11. In the definitive expression of matrices, one shall take into account the following parameters:

$$
b_{11}=1, a_{11}=\frac{2 h}{15}+m
$$

Dissipativity of the method. In this paragraph, we demonstrate the dissipativity of the semi discrete scheme. Consider the following function:

$$
\hat{E}(t ; y):=\frac{1}{2}\left[\int_{0}^{1} y_{t}(x, t)^{2} d x+\int_{0}^{1} y_{x}(x, t)^{2} d x+m y_{t}(0, t)^{2}\right]
$$

where $y \in \mathcal{C}^{2}([0, \infty) ; V)$.

Theorem 12. The solution $y_{h}$ of the problem $G^{h}$ satisfies:

$$
\forall t>0, \frac{d}{d t} \hat{E}\left(t ; y_{h}\right)=-\beta\left(y_{h}\right)_{t}^{2}(0) \leq 0 .
$$

Proof. For all $y_{h} \in C^{2}\left([0, \infty) ; V_{h}\right)$, we have:

$$
\hat{E}\left(t ; y_{h}\right)=\frac{1}{2}\left[\int_{0}^{1}\left(\left(y_{h}\right)_{t}^{2}(x, t)+\left(y_{h}\right)_{x}(x, t)^{2}\right) d x+m\left(y_{h}\right)_{t}(0, t)^{2}\right] .
$$


Then we have:

$$
\frac{d}{d t} \hat{E}\left(t ; y_{h}\right)=\int_{0}^{1}\left(y_{h}\right)_{x}\left(y_{h}\right)_{t x} d x+\int_{0}^{1}\left(y_{h}\right)_{t}\left(y_{h}\right)_{t t} d x+m\left(y_{h}\right)_{t}(0)\left(y_{h}\right)_{t t}(0)
$$

In other part, by replacing $w_{h}$ by $\left(y_{h}\right)_{t}$ in (51), one obtains:

$$
\int_{0}^{1}\left(y_{h}\right)_{t t}\left(y_{h}\right)_{t} d x+\int_{0}^{1}\left(y_{h}\right)_{t x}\left(y_{h}\right)_{x} d x=-m\left(y_{h}\right)_{t t}(0)\left(y_{h}\right)_{t}(0)-\beta\left(y_{h}\right)_{t}^{2}(0) .
$$

Then we obtain $\frac{d}{d t} \hat{E}\left(t ; y_{h}\right)=-\beta\left(y_{h}\right)_{t}^{2}(0)$.

\subsubsection{Fully discrete scheme}

Consider a new variable $z_{h}:=\left(y_{h}\right)_{t}$. Rewriting (54) as a first order ODE, we get:

$$
\begin{array}{r}
\mathcal{N} \mathbb{U}_{t}=\mathcal{M U} \\
\mathbb{U}_{0}=\left[\mathbb{Y}_{0} \mathbb{Z}_{0}\right]^{\top},
\end{array}
$$

where $\mathbb{Z}:=\mathbb{Y}_{t}=\left[\begin{array}{llll}Z_{1} & Z_{2} & \ldots & Z_{2 p}\end{array}\right]^{\top}$ be its representation in the basis $\left\{\phi_{i}\right\}_{i}$, $\mathbb{U}=\left[\mathbb{Y} \mathbb{Z}^{\top}\right.$,

$$
\mathcal{N}=\left[\begin{array}{cc}
\mathbb{I}_{2 p} & \mathbb{O}_{2 p} \\
\mathbb{O}_{2 p} & \mathbb{A}
\end{array}\right] \quad \text { and } \quad \mathcal{M}=\left[\begin{array}{cc}
\mathbb{O}_{2 p} & \mathbb{I}_{2 p} \\
-\mathbb{K} & -\beta \mathbb{B}
\end{array}\right]
$$

The time interval is discretized into $s$ equidistant subintervals, for a fixed $s \in \mathbb{N}$. Let $\Delta t:=T / s$ denote the time step and

$$
t_{k}=k \Delta t, \quad \forall k \in\{0,1, \ldots, s\},
$$

the nodes of the discretization.

We adopt as notation $U_{h}^{k}=\left[y_{h}^{k} z_{h}^{k}\right]^{\top}$, the approximation of the solution $U_{h}$ at time $t_{k}$. Let $\mathbb{Y}_{k}$ and $\mathbb{Z}_{k}$ be the vector representations of $y_{h}^{k}$ and $z_{h}^{k}$ in the considered basis in $V_{h}$.

Applying the Crank-Nicholson scheme to the system (61), we obtain:

$$
\begin{gathered}
\mathbb{M} \mathbb{U}_{k+1}=\mathbb{S} \mathbb{U}_{k}, \\
\mathbb{U}_{0}=\left[\begin{array}{ll}
\mathbb{Y}_{0} & \mathbb{Z}_{0}
\end{array}\right]^{\top},
\end{gathered}
$$

for all $k=0,1, \ldots, s-1$, with

$$
\mathbb{M}=\frac{\mathcal{N}}{\Delta t}-\frac{\mathcal{M}}{2}=\left[\begin{array}{lc}
\frac{\mathbb{I}_{2 p}}{\Delta t} & -\frac{\mathbb{I}_{2 p}}{2} \\
\frac{\mathbb{K}}{2} & \frac{\mathbb{A}}{\Delta t}+\beta \frac{\mathbb{B}}{2}
\end{array}\right]
$$


and

$$
\mathbb{S}=\frac{\mathcal{N}}{\Delta t}+\frac{\mathcal{M}}{2}=\left[\begin{array}{cc}
\frac{\mathbb{I}_{2 p}}{\Delta t} & \frac{\mathbb{I}_{2 p}}{2} \\
-\frac{\mathbb{K}}{2} & \frac{\mathbb{A}}{\Delta t}-\beta \frac{\mathbb{B}}{2}
\end{array}\right] .
$$

Moreover, for all $k=0,1, \ldots, s-1$,

$$
\begin{aligned}
\frac{\mathbb{Y}_{k+1}-\mathbb{Y}_{k}}{\Delta t} & =\frac{\mathbb{Z}_{k+1}+\mathbb{Z}_{k}}{2}, \\
\mathbb{A} \frac{\mathbb{Z}_{k+1}-\mathbb{Z}_{k}}{\Delta t} & =-\mathbb{K} \frac{\mathbb{Y}_{k+1}+\mathbb{Y}_{k}}{2}-\beta \mathbb{B} \frac{\mathbb{Z}_{k+1}+\mathbb{Z}_{k}}{2} .
\end{aligned}
$$

Dissipativity of the method. In the following, we show that the scheme (65)-(66) dissipates the norm. The natural norm of $U_{h}=U_{h}(t)=\left[y_{h} z_{h}\right]^{\top}$, the solution of the semi-discretized system, is defined as:

$$
\left\|U_{h}\right\|^{2}=\frac{1}{2}\left[\int_{0}^{1}\left(y_{h}\right)_{x}^{2} d x+\int_{0}^{1} z_{h}^{2} d x+m z_{h}(0)^{2}\right] .
$$

We have the following theorem:

Theorem 13. For all $k \in \mathbb{N}$, we have:

$$
\left\|U_{h}^{k+1}\right\|^{2}-\left\|U_{h}^{k}\right\|^{2}=-\beta \Delta t\left(\frac{z_{h}^{k+1}(0)+z_{h}^{k}(0)}{2}\right)^{2} \leq 0 .
$$

Proof. We have:

$$
\begin{aligned}
& \left\|U_{h}^{k+1}\right\|^{2}-\left\|U_{h}^{k}\right\|^{2} \\
& =\frac{1}{2}\left[\int_{0}^{1}\left(\left(\left(y_{h}\right)_{x}^{k+1}\right)^{2}-\left(\left(y_{h}\right)_{x}^{k}\right)^{2}\right) d x+\int_{0}^{1}\left(\left(z_{h}^{k+1}\right)^{2}\right.\right. \\
& \left.\left.\quad-\left(z_{h}^{k}\right)^{2}\right) d x+m\left(\left(z_{h}^{k+1}\right)^{2}(0)-\left(z_{h}^{k}\right)^{2}(0)\right)\right]
\end{aligned}
$$

Using Crank-Nicholson scheme, one obtains:

$$
\frac{y_{h}^{k+1}-y_{h}^{k}}{\Delta t}=\frac{z_{h}^{k+1}+z_{h}^{k}}{2} .
$$

Multiplying (69) by $z_{h}^{k+1}-z_{h}^{k}$ and integrating it over [0;1], we have:

$$
\frac{1}{2} \int_{0}^{1}\left(\left(z_{h}^{k+1}\right)^{2}-\left(z_{h}^{k}\right)^{2}\right) d x=\int_{0}^{1} \frac{y_{h}^{k+1}-y_{h}^{k}}{\Delta t}\left(z_{h}^{k+1}-z_{h}^{k}\right) d x .
$$


Moreover, using Crank-Nicholson scheme on (11), we obtain:

$$
\begin{array}{r}
\int_{0}^{1}\left(\frac{z_{h}^{k+1}-z_{h}^{k}}{\Delta t} w_{h}+\frac{\left(y_{h}^{k+1}\right)_{x}+\left(y_{h}^{k}\right)_{x}}{2}\left(w_{h}\right)_{x}\right) d x \\
+\beta \frac{z_{h}^{k+1}(0)+z_{h}^{k}(0)}{2} w_{h}(0)+m \frac{z_{h}^{k+1}(0)-z_{h}^{k}(0)}{\Delta t} w_{h}(0)=0 .
\end{array}
$$

Substituting $w_{h}$ by $y_{h}^{k+1}$ and next by $y_{h}^{k}$ in (71), one has:

$$
\begin{array}{r}
\frac{1}{2} \int_{0}^{1}\left(\left(y_{h}\right)_{x}^{k+1}\right)^{2} d x=-\frac{1}{2} \int_{0}^{1}\left(y_{h}\right)_{x}^{k+1}\left(y_{h}\right)_{x}^{k} d x-\int_{0}^{1} \frac{z_{h}^{k+1}-z_{h}^{k}}{\Delta t} y_{h}^{k+1} d x \\
-m \frac{z_{h}^{k+1}(0)-z_{h}^{k}(0)}{\Delta t} y_{h}^{k+1}(0)-\beta \frac{z_{h}^{k+1}(0)+z_{h}^{k}(0)}{2} y_{h}^{k+1}(0), \\
\frac{1}{2} \int_{0}^{1}\left(\left(y_{h}\right)_{x}^{k}\right)^{2} d x=-\frac{1}{2} \int_{0}^{1}\left(y_{h}\right)_{x}^{k+1}\left(y_{h}\right)_{x}^{k} d x-\int_{0}^{1} \frac{z_{h}^{k+1}-z_{h}^{k}}{\Delta t} y_{h}^{k} d x \\
-m \frac{z_{h}^{k+1}(0)-z_{h}^{k}(0)}{\Delta t} y_{h}^{k}(0)-\beta \frac{z_{h}^{k+1}(0)+z_{h}^{k}(0)}{2} y_{h}^{k}(0) .
\end{array}
$$

Substracting (73) from (72) yields:

$$
\begin{aligned}
\frac{1}{2} \int_{0}^{1} & \left.\left.\left(\left(y_{h}\right)_{x}^{k+1}\right)^{2}-\left(y_{h}\right)_{x}^{k}\right)^{2}\right) d x \\
= & -\frac{1}{2} \int_{0}^{1}\left(\left(z_{h}^{k+1}\right)^{2}-\left(z_{h}^{k}\right)^{2}\right) d x-\frac{m}{2}\left(z_{h}^{k+1}(0)^{2}-z_{h}^{k}(0)^{2}\right) \\
& -\beta \frac{\Delta t}{4}\left(z_{h}^{k+1}(0)+z_{h}^{k}(0)\right)^{2} .
\end{aligned}
$$

Finally:

$$
\left\|U_{h}^{k+1}\right\|^{2}-\left\|U_{h}^{k}\right\|^{2}=-\beta \Delta t\left(\frac{z_{h}^{k+1}(0)+z_{h}^{k}(0)}{2}\right)^{2} .
$$




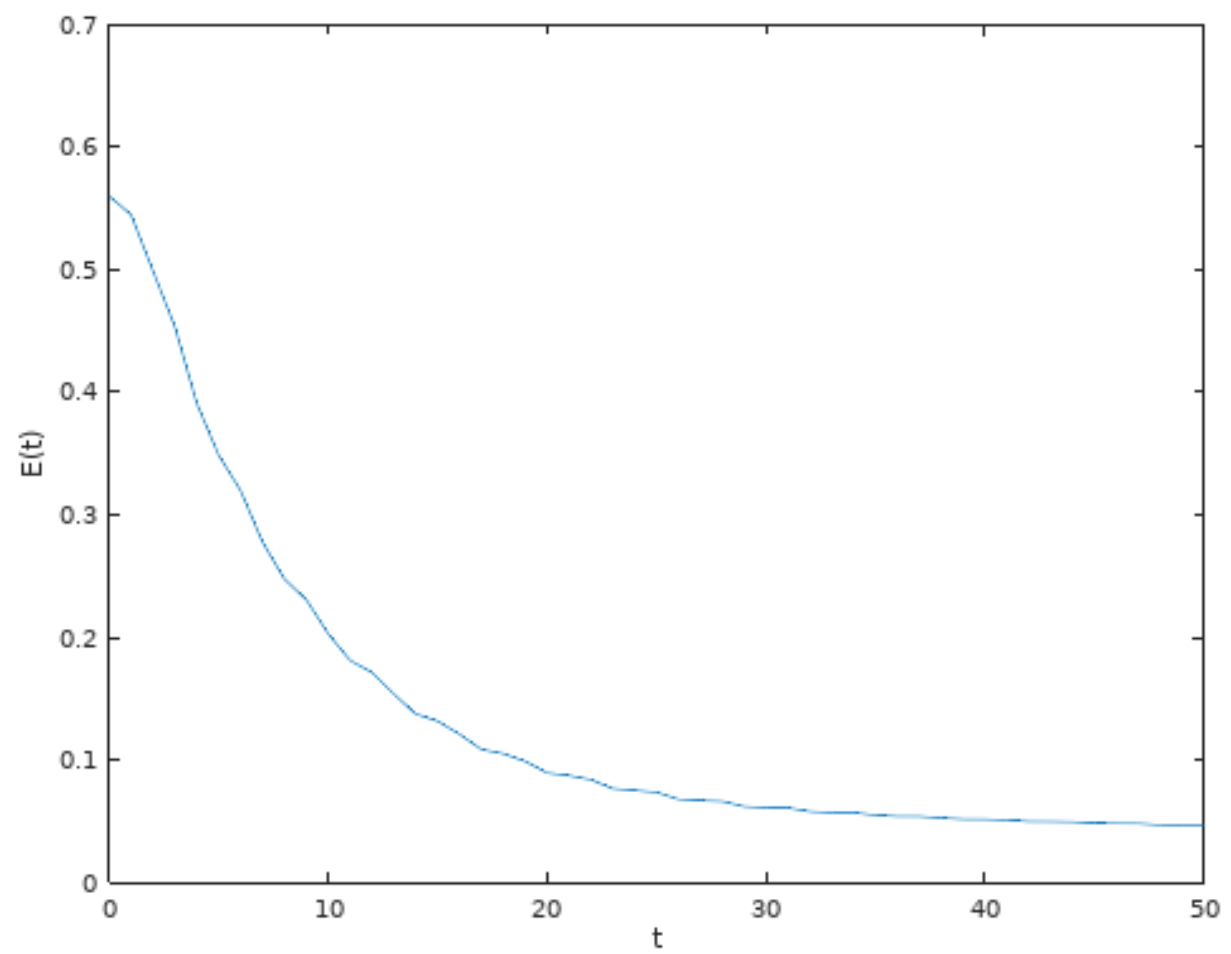

Figure 1: Energy dissipation $E(t)$

\section{Errors estimates}

\subsection{A-priori error estimates for the semi-discrete scheme}

In this paragraph, we derive the a-priori estimates for the Galerkin solution of (51)-(52), where the discrete space $V_{h}$ is the space of $\mathbb{P}_{2}$ Lagrange polynomials. These estimates are based on the method used in Choo et al. [18]. The Lagrange interpolation of the weak solution $y \in V_{h}$ is denoted by $\tilde{y}$ :

$$
\tilde{y}(x, t)=\sum_{j=0}^{p-1}\left(y\left(x_{j}, t\right) \phi_{2 j+1}(x)+y\left(x_{j+\frac{1}{2}}, t\right) \phi_{2 j+2}(x)\right) .
$$

Suppose that

$$
y \in C\left([0, T] ; H_{E}^{2}(0,1)\right), y_{t} \in L^{2}\left(0, T ; H_{E}^{2}(0,1)\right), y_{t t} \in L^{2}\left(0, T ; H_{E}^{1}(0,1)\right) .
$$


Then for almost every $t$, we have (cf. Brenner et al. [3], Choo et al. [18]):

$$
\begin{aligned}
& \|y-\tilde{y}\|_{H^{1}(0,1)} \leq C h\|y\|_{H^{2}(0,1)}, \\
& \left\|y_{t}-\tilde{y}_{t}\right\|_{H^{1}(0,1)} \leq C h\left\|y_{t}\right\|_{H^{2}(0,1)}, \\
& \left\|y_{t t}-\tilde{y}_{t t}\right\|_{L^{2}(0,1)} \leq C h\left\|y_{t t}\right\|_{H^{1}(0,1)} .
\end{aligned}
$$

The error of the semi-discrete solution $y_{h}$ is defined as $\epsilon^{h}:=y_{h}-\tilde{y} \in V_{h}$. Using (51), it follows:

$$
\begin{aligned}
\int_{0}^{1} \epsilon_{t t}^{h} w d x & +\int_{0}^{1} \epsilon_{x}^{h} w_{x} d x+m \epsilon_{t t}^{h}(0) w(0)+\beta \epsilon_{t}^{h}(0) w(0) \\
& =\int_{0}^{1}\left(y_{t t}-\tilde{y}_{t t}\right) w d x+\int_{0}^{1}\left(y_{x}-\tilde{y}_{x}\right) w_{x} d x
\end{aligned}
$$

for all $w \in V_{h}, \quad t>0$. Using $w=\epsilon_{t}^{h}$, we obtain:

$$
\begin{gathered}
\int_{0}^{1} \epsilon_{t t}^{h} \epsilon_{t}^{h} d x+\int_{0}^{1} \epsilon_{x}^{h} \epsilon_{t x}^{h} d x=\int_{0}^{1}\left(y_{t t}-\tilde{y}_{t t}\right) \epsilon_{t}^{h} d x+\int_{0}^{1}\left(y_{x}-\tilde{y}_{x}\right) \epsilon_{t x}^{h} d x \\
-\beta \epsilon_{t}^{h}(0)^{2}-m \epsilon_{t t}^{h}(0) \epsilon_{t}^{h}(0) \\
\leq \int_{0}^{1}\left(y_{t t}-\tilde{y}_{t t}\right) \epsilon_{t}^{h} d x+\int_{0}^{1}\left(y_{x}-\tilde{y}_{x}\right) \epsilon_{t x}^{h} d x \\
\quad-m \epsilon_{t t}^{h}(0) \epsilon_{t}^{h}(0)
\end{gathered}
$$

for almost every $t \in[0, T]$. Then, for almost every $t \in[0, T]$ we have:

$$
\begin{aligned}
\frac{d}{d t} \hat{E}\left(t ; \epsilon^{h}\right) & =\int_{0}^{1}\left(y_{t t}-\tilde{y}_{t t}\right) \epsilon_{t}^{h} d x+\int_{0}^{1}\left(y_{x}-\tilde{y}_{x}\right) \epsilon_{t x}^{h} d x-\beta \epsilon_{t}^{h}(0)^{2} \\
& \leq \int_{0}^{1}\left(y_{t t}-\tilde{y}_{t t}\right) \epsilon_{t}^{h} d x+\int_{0}^{1}\left(y_{x}-\tilde{y}_{x}\right) \epsilon_{t x}^{h} d x .
\end{aligned}
$$

Integrating the previous expression over $[0, t]$. It follows:

$$
\hat{E}\left(t ; \epsilon^{h}\right) \leq \hat{E}\left(0 ; \epsilon^{h}(0)\right)+\int_{0}^{t}\left(\int_{0}^{1}\left(y_{t t}-\tilde{y}_{t t}\right) \epsilon_{t}^{h} d x+\int_{0}^{1}\left(y_{x}-\tilde{y}_{x}\right) \epsilon_{t x}^{h} d x\right) d s .
$$

After performing partial integration, one has:

$$
\begin{aligned}
& \hat{E}\left(t ; \epsilon^{h}\right) \leq \hat{E}\left(0 ; \epsilon^{h}(0)\right)+\int_{0}^{t}\left(\int_{0}^{1}\left(y_{t t}-\tilde{y}_{t t}\right) \epsilon_{t}^{h} d x-\int_{0}^{1} \epsilon_{x}^{h}\left(y_{t x}-\tilde{y}_{t x}\right) d x\right) d s \\
& +\int_{0}^{1}\left(y_{x}(x, t)-\tilde{y}_{x}(x, t)\right) \epsilon_{x}^{h}(x, t) d x-\int_{0}^{1}\left(y_{x}(x, 0)-\tilde{y}_{x}(x, 0)\right) \epsilon_{x}^{h}(x, 0) d x .
\end{aligned}
$$


Applying the Cauchy-Schwarz and Young inequalities to the second member of the previous inequality, we obtain for all $\eta>0$ :

$$
\begin{aligned}
\hat{E}\left(t ; \epsilon^{h}\right) \leq \hat{E}\left(0 ; \epsilon^{h}(0)\right)+\eta\left(\left\|y_{t t}-\tilde{y}_{t t}\right\|_{L^{2}\left(0, T ; L^{2}(0,1)\right)}^{2}+\left\|y_{t}-\tilde{y}_{t}\right\|_{L^{2}\left(0, T ; H^{1}(0,1)\right)}^{2}\right. \\
\left.+\left\|y_{x}(., t)-\tilde{y}_{x}(., t)\right\|_{L^{2}(0,1)}^{2}+\left\|y_{x}(., 0)-\tilde{y}_{x}(., 0)\right\|_{L^{2}(0,1)}^{2}\right) \\
+\frac{1}{4 \eta}\left(\int_{0}^{t} \hat{E}\left(s ; \epsilon^{h}(s)\right) d s+\hat{E}\left(t ; \epsilon^{h}(t)\right)+\hat{E}\left(0 ; \epsilon^{h}(0)\right)\right) .
\end{aligned}
$$

This leads to:

$$
\begin{aligned}
& \left(1-\frac{1}{4 \eta}\right) \hat{E}\left(t ; \epsilon^{h}(t)\right) \leq\left(1+\frac{1}{4 \eta}\right) \hat{E}\left(0 ; \epsilon^{h}(0)\right) \\
& \quad+\frac{1}{4 \eta} \int_{0}^{t} \hat{E}\left(s ; \epsilon^{h}(s)\right) d s+\eta\left(\left\|y_{t t}-\tilde{y}_{t t}\right\|_{L^{2}\left(0, T ; L^{2}(0,1)\right)}^{2}\right. \\
& \left.\quad+\left\|y_{t}-\tilde{y}_{t}\right\|_{L^{2}\left(0, T ; H^{1}(0,1)\right)}^{2}+2\|y-\tilde{y}\|_{C\left([0, T] ; H^{1}(0,1)\right)}^{2}\right) .
\end{aligned}
$$

Take $\eta=1$. Using (76), we obtain:

$$
\begin{aligned}
& \hat{E}\left(t ; \epsilon^{h}(t)\right) \leq \frac{5}{3} \hat{E}\left(0 ; \epsilon^{h}(0)\right)+\frac{1}{3} \int_{0}^{t} \hat{E}\left(s ; \epsilon^{h}(s)\right) d s \\
& \quad+C h^{2}\left(\left\|y_{t t}\right\|_{L^{2}\left(0, T ; H^{1}(0,1)\right)}^{2}+\left\|y_{t}\right\|_{L^{2}\left(0, T ; H^{2}(0,1)\right)}^{2}+\|y\|_{C\left([0, T] ; H^{2}(0,1)\right)}^{2}\right)
\end{aligned}
$$

Applying the Gronwall inequality to (79), one obtains:

$$
\begin{aligned}
\hat{E}\left(t ; \epsilon^{h}(t)\right) \leq & C\left[\hat{E}\left(0 ; \epsilon^{h}(0)\right)+h^{2}\left(\left\|y_{t t}\right\|_{L^{2}\left(0, T ; H^{1}(0,1)\right)}^{2}+\left\|y_{t}\right\|_{L^{2}\left(0, T ; H^{2}(0,1)\right)}^{2}\right.\right. \\
& \left.\left.+\|y\|_{C\left([0, T] ; H^{2}(0,1)\right)}^{2}\right)\right] .
\end{aligned}
$$

Finally, we have the following result:

Theorem 14. Suppose (75), and take $V_{h}$ the space of the piecewise $\mathbb{P}_{2}$ Lagrange polynomials. For $y_{h} \in C^{2}\left([0, T] ; V_{h}\right)$ solving (51)-(52), we have:

$$
\hat{E}\left(t ; y_{h}-y\right)^{\frac{1}{2}} \leq C\left[\hat{E}\left(0 ; \epsilon^{h}(0)\right)^{\frac{1}{2}}+h\left(\left\|y_{t t}\right\|_{L^{2}\left(0, T ; H^{1}(0,1)\right)}\right.\right.
$$




$$
\left.\left.+\left\|y_{t}\right\|_{L^{2}\left(0, T ; H^{2}(0,1)\right)}+\|y\|_{C\left([0, T] ; H^{2}(0,1)\right)}\right)\right] .
$$

In addition, if $y_{h}^{0}$ and $y_{h}^{1}$ are Lagrange interpolations of $y_{0}$ and $y_{1}$, then we have:

$$
\begin{aligned}
\hat{E}\left(t ; y_{h}-y\right)^{\frac{1}{2}} \leq & C h\left(\left\|y_{t t}\right\|_{L^{2}\left(0, T ; H^{1}(0,1)\right)}+\left\|y_{t}\right\|_{L^{2}\left(0, T ; H^{2}(0,1)\right)}\right. \\
& \left.+\|y\|_{C\left([0, T] ; H^{2}(0,1)\right)}\right) .
\end{aligned}
$$

\subsection{A-priori error estimates for the fully discrete scheme}

In this paragraph, a-priori error estimates are given for the scheme (65)-(66). Assume that $y \in H^{4}\left(0, T ; H_{E}^{1}(0,1)\right)$. Let $\breve{y} \in V_{h}$ be the projection of the weak solution $y$, such that:

$$
a\left(y(t)-\breve{y}(t), w_{h}\right)=0, \quad \forall w_{h} \in V_{h},
$$

for all $t \in[0 ; T]$. One verifies that $\breve{y} \in H^{4}\left(0, T ; H_{E}^{1}(0,1)\right)$ because the projection $y \mapsto \breve{y}$ is bounded in $H_{E}^{1}(0,1)$. Moreover, let $y^{e}:=y-\breve{y}$ be the error of the projection. Suppose that $y \in H^{2}\left(0, T ; H_{E}^{2}(0,1)\right)$. We have (cf. Strang et al. $[6])$ :

$$
\begin{array}{r}
\left\|y^{e}\right\|_{H^{1}(0,1)} \leq C h\|y\|_{H^{2}(0,1)}, \\
\left\|y_{t}^{e}\right\|_{H^{1}(0,1)} \leq C h\left\|y_{t}\right\|_{H^{2}(0,1)}, \\
\left\|y_{t t}^{e}\right\|_{H^{1}(0,1)} \leq C h\left\|y_{t t}\right\|_{H^{2}(0,1)} .
\end{array}
$$

Let $U\left(t_{k}\right)=\left(y\left(t_{k}\right) ; y_{t}\left(t_{k}\right)\right)^{\top}$ denote the weak solution of (14) at time $t_{k}$ and $U^{k}=\left(y^{k} ; z^{k}\right)$ the $k$-th iteration of the fully discrete scheme (65)-(66) approximating $U\left(t_{k}\right)$. Then the approximating error is defined by:

$$
\Psi^{k}:=y^{k}-\breve{y}\left(t_{k}\right), \quad \Phi^{k}:=z^{k}-\breve{y}_{t}\left(t_{k}\right),
$$

and $U_{e}^{k}:=\left(\Psi^{k} ; \Phi^{k}\right)$, for all $k \in\{0,1, \ldots, s\}$.

Theorem 15. Suppose that

$$
y \in H^{2}\left(0, T ; H_{E}^{2}(0,1)\right) \cap H^{4}\left(0, T ; H_{E}^{1}(0,1)\right) .
$$

Let $k \in\{1, \ldots, s\}$. Then we have:

$$
\begin{aligned}
\left\|U^{k}-U\left(t_{k}\right)\right\| \leq & C\left[\left\|U_{e}^{0}\right\|+h\|y\|_{H^{2}\left(0, T ; H^{2}(0,1)\right)}\right. \\
& \left.+(\Delta t)^{\frac{3}{2}}\left(\left\|y_{t t}\right\|_{H^{2}\left(0, T ; H^{1}(0,1)\right)}+\left\|y_{t t}\right\|_{L^{2}\left(0, T ; H^{2}(0,1)\right)}\right)\right] .
\end{aligned}
$$


Proof. Let $k \in\{0,1, \ldots, s\}$. The Taylor theorem yields:

$$
\forall x \in[0,1], \frac{\breve{y}\left(x, t_{k+1}\right)-\breve{y}\left(x, t_{k}\right)}{\Delta t}=\frac{\breve{y}_{t}\left(x, t_{k+1}\right)+\breve{y}_{t}\left(x, t_{k}\right)}{2}+\Delta t T_{1}^{k}(x),
$$

with

$$
\begin{aligned}
T_{1}^{k}(x)= & \int_{t_{k+\frac{1}{2}}}^{t_{k+1}} \frac{\breve{y}_{t t t}(x, t)}{2(\Delta t)^{2}}\left(t_{k+1}-t\right)^{2} d t+\int_{t_{k}}^{t_{k+\frac{1}{2}}} \frac{\breve{y}_{t t t}(x, t)}{2(\Delta t)^{2}}\left(t_{k}-t\right)^{2} d t \\
& -\int_{t_{k+\frac{1}{2}}}^{t_{k+1}} \frac{\breve{y}_{t t t}(x, t)}{2 \Delta t}\left(t_{k+1}-t\right) d t+\int_{t_{k}}^{t_{k+\frac{1}{2}}} \frac{\breve{y}_{t t t}(x, t)}{2 \Delta t}\left(t_{k}-t\right) d t .
\end{aligned}
$$

From (84), one verifies:

$$
\frac{\Psi^{k+1}-\Psi^{k}}{\Delta t}+\Delta t T_{1}^{k}=\frac{\Phi^{k+1}+\Phi^{k}}{2}
$$

Multiplying the previous expression by $\left(\Phi^{k+1}-\Phi^{k}\right)$ and integrating it over $[0,1]$ yields:

$$
\begin{aligned}
\int_{0}^{1} \frac{\Psi^{k+1}-\Psi^{k}}{\Delta t}\left(\Phi^{k+1}-\Phi^{k}\right) d x= & \frac{1}{2} \int_{0}^{1}\left(\left(\Phi^{k+1}\right)^{2}-\left(\Phi^{k}\right)^{2}\right) d x \\
& -\Delta t \int_{0}^{1} T_{1}^{k}(x)\left(\Phi^{k+1}-\Phi^{k}\right) d x .
\end{aligned}
$$

In addition, substituting $t$ by $t_{k+\frac{1}{2}}$ in (11) and using the Taylor expansion, we obtain:

$$
\begin{aligned}
& \int_{0}^{1} \frac{y_{t}\left(x, t_{k+1}\right)-y_{t}\left(x, t_{k}\right)}{\Delta t} w(x) d x+\int_{0}^{1} \frac{y_{x}\left(x, t_{k+1}\right)+y_{x}\left(x, t_{k}\right)}{2} w_{x}(x) d x \\
+ & \left(m \frac{y_{t}\left(0, t_{k+1}\right)-y_{t}\left(0, t_{k}\right)}{\Delta t}+\beta \frac{y_{t}\left(0, t_{k+1}\right)+y_{t}\left(0, t_{k}\right)}{2}\right) w(0)=\Delta t T_{2}^{k}(w),
\end{aligned}
$$

where the functional $T_{2}^{k}: H_{E}^{1}(0,1) \rightarrow \mathbb{R}$ is defined by:

$$
\begin{gathered}
T_{2}^{k}(w)=\int_{0}^{1}\left(\int_{t_{k+\frac{1}{2}}}^{t_{k+1}} \frac{y_{t t t t}(x, t)}{2(\Delta t)^{2}}\left(t_{k+1}-t\right)^{2} d t\right. \\
\left.+\int_{t_{k}}^{t_{k+\frac{1}{2}}} \frac{y_{t t t t}(x, t)}{2(\Delta t)^{2}}\left(t_{k}-t\right)^{2} d t\right) w(x) d x \\
+\int_{0}^{1}\left(\int_{t_{k+\frac{1}{2}}}^{t_{k+1}} \frac{y_{t t x}(x, t)}{2 \Delta t}\left(t_{k+1}-t\right) d t-\int_{t_{k}}^{t_{k+\frac{1}{2}}} \frac{y_{t t x}(x, t)}{2 \Delta t}\left(t_{k}-t\right) d t\right) w_{x}(x) d x
\end{gathered}
$$




$$
\begin{aligned}
+ & m\left(\int_{t_{k+\frac{1}{2}}}^{t_{k+1}} \frac{y_{t t t t}(0, t)}{2(\Delta t)^{2}}\left(t_{k+1}-t\right)^{2} d t+\int_{t_{k}}^{t_{k+\frac{1}{2}}} \frac{y_{t t t t}(0, t)}{2(\Delta t)^{2}}\left(t_{k}-t\right)^{2} d t\right) w(0) \\
& +\beta\left(\int_{t_{k+\frac{1}{2}}}^{t_{k+1}} \frac{y_{t t t}(0, t)}{2 \Delta t}\left(t_{k+1}-t\right) d t-\int_{t_{k}}^{t_{k+\frac{1}{2}}} \frac{y_{t t t}(0, t)}{2 \Delta t}\left(t_{k}-t\right) d t\right) w(0) .
\end{aligned}
$$

Now, from (66) and (86), one has for all $w_{h} \in V_{h}$ :

$$
\begin{aligned}
\int_{0}^{1} \frac{\Phi^{k+1}-\Phi^{k}}{\Delta t} w_{h} d x+\int_{0}^{1} \frac{\Psi_{x}^{k+1}+\Psi_{x}^{k}}{2}\left(w_{h}\right)_{x} d x+\left(m \frac{\Phi^{k+1}(0)-\Phi^{k}(0)}{\Delta t}\right. \\
\left.+\beta \frac{\Phi^{k+1}(0)+\Phi^{k}(0)}{2}\right) w_{h}(0)=G^{k}\left(w_{h}\right)-\Delta t T_{2}^{k}\left(w_{h}\right),
\end{aligned}
$$

where the functional $G^{k}\left(w_{h}\right)$ is defined as:

$$
\begin{aligned}
G^{k}\left(w_{h}\right):= & \int_{0}^{1} \frac{y_{t}^{e}\left(x, t_{k+1}\right)-y_{t}^{e}\left(x, t_{k}\right)}{\Delta t} w_{h} d x \\
& +\left(m \frac{y_{t}^{e}\left(0, t_{k+1}\right)-y_{t}^{e}\left(0, t_{k}\right)}{\Delta t}+\beta \frac{y_{t}^{e}\left(0, t_{k+1}\right)+y_{t}^{e}\left(0, t_{k}\right)}{2}\right) w_{h}(0) .
\end{aligned}
$$

Taking $w_{h}=\frac{\Delta t}{2}\left(\Phi^{k+1}+\Phi^{k}\right) \in V_{h}$ in (88), yields:

$$
\begin{array}{r}
\frac{1}{2}\left(\int_{0}^{1}\left(\left(\Phi^{k+1}\right)^{2}-\left(\Phi^{k}\right)\right)^{2} d x\right)=-\frac{\Delta t}{2} \int_{0}^{1}\left(\frac{\Psi_{x}^{k+1}+\Psi_{x}^{k}}{2}\right)\left(\Phi_{x}^{k+1}+\Phi_{x}^{k}\right) d x \\
-\frac{\Delta t}{2}\left(\Phi^{k+1}(0)+\Phi^{k}(0)\right)\left(m \frac{\Phi^{k+1}(0)-\Phi^{k}(0)}{\Delta t}+\beta \frac{\Phi^{k+1}(0)+\Phi^{k}(0)}{2}\right) \\
+\frac{\Delta t}{2} G^{k}\left(\Phi^{k+1}+\Phi^{k}\right)-\frac{(\Delta t)^{2}}{2} T_{2}^{k}\left(\Phi^{k+1}+\Phi^{k}\right) .
\end{array}
$$

Then we have:

$$
\begin{aligned}
\left\|U_{e}^{k+1}\right\|^{2}-\left\|U_{e}^{k}\right\|^{2}=\frac{1}{2} \int_{0}^{1}\left(\left(\Psi_{x}^{k+1}\right)^{2}-\left(\Psi_{x}^{k}\right)^{2}\right) d x \\
\quad-\frac{\Delta t}{2} \int_{0}^{1} \frac{\Psi_{x}^{k+1}+\Psi^{k}}{2}\left(\Phi_{x}^{k+1}+\Phi_{x}^{k}\right) d x-\beta \frac{\Delta t}{4}\left(\Phi^{k+1}(0)+\Phi^{k}(0)\right)^{2} \\
\quad+\frac{\Delta t}{2} G^{k}\left(\Phi^{k+1}+\Phi^{k}\right)-\frac{(\Delta t)^{2}}{2} T_{2}^{k}\left(\Phi^{k+1}+\Phi^{k}\right) \\
\leq \frac{1}{2} \int_{0}^{1}\left(\left(\Psi_{x}^{k+1}\right)^{2}-\left(\Psi_{x}^{k}\right)^{2}\right) d x-\frac{\Delta t}{2} \int_{0}^{1} \frac{\Psi_{x}^{k+1}+\Psi^{k}}{2}\left(\Phi_{x}^{k+1}+\Phi_{x}^{k}\right) d x
\end{aligned}
$$




$$
+\frac{\Delta t}{2} G^{k}\left(\Phi^{k+1}+\Phi^{k}\right)-\frac{(\Delta t)^{2}}{2} T_{2}^{k}\left(\Phi^{k+1}+\Phi^{k}\right) .
$$

Now by (85), we obtain:

$$
\begin{gathered}
\int_{0}^{1} \frac{\Psi_{x}^{k+1}+\Psi^{k}}{2}\left(\Phi_{x}^{k+1}+\Phi_{x}^{k}\right) d x \\
=\int_{0}^{1} \frac{\left(\Psi_{x}^{k+1}\right)^{2}-\left(\Psi_{x}^{k}\right)^{2}}{\Delta t} d x+\Delta t \int_{0}^{1}\left(T_{1}^{k}\right)_{x}\left(\Psi_{x}^{k+1}+\Psi_{x}^{k}\right) d x .
\end{gathered}
$$

Then we get:

$$
\begin{aligned}
\left\|U_{e}^{k+1}\right\|^{2}-\left\|U_{e}^{k}\right\|^{2} \leq- & \frac{(\Delta t)^{2}}{2} \int_{0}^{1}\left(T_{1}^{k}\right)_{x}\left(\Psi_{x}^{k+1}+\Psi_{x}^{k}\right) d x \\
& +\frac{\Delta t}{2} G^{k}\left(\Phi^{k+1}+\Phi^{k}\right)-\frac{(\Delta t)^{2}}{2} T_{2}^{k}\left(\Phi^{k+1}+\Phi^{k}\right)
\end{aligned}
$$

It can easily be seen that:

$$
\left\|T_{1}^{k}\right\|_{H^{1}(0,1)}^{2} \leq \Delta t \int_{t_{k}}^{t_{k+1}}\left\|\breve{y}_{t t t}(t)\right\|_{H^{1}(0,1)}^{2} d t \leq C \Delta t \int_{t_{k}}^{t_{k+1}}\left\|y_{t t t}(t)\right\|_{H^{1}(0,1)}^{2} d t .
$$

Rewriting the second term of $T_{2}^{k}(w)$ (using the fact $w(1)=0$ ), one obtains:

$$
\begin{aligned}
\int_{0}^{1} & \left(\int_{t_{k+\frac{1}{2}}}^{t_{k+1}} \frac{y_{t t x}(x, t)}{2 \Delta t}\left(t_{k+1}-t\right) d t-\int_{t_{k}}^{t_{k+\frac{1}{2}}} \frac{y_{t t x}(x, t)}{2 \Delta t}\left(t_{k}-t\right) d t\right) w_{x}(x) d x \\
& =\int_{t_{k}}^{t_{k+\frac{1}{2}}} \frac{t_{k}-t}{2 \Delta t}\left[y_{t t x}(0, t) w(0)+\int_{0}^{1} y_{t t x x}(x, t) w(x) d x\right] d t \\
& -\int_{t_{k+\frac{1}{2}}}^{t_{k+1}} \frac{t_{k+1}-t}{2 \Delta t}\left[y_{t t x}(0, t) w(0)+\int_{0}^{1} y_{t t x x}(x, t) w(x) d x\right] d t
\end{aligned}
$$

Then we have:

$$
\begin{aligned}
T_{2}^{k}\left(\Phi^{k}\right) \leq & C\left[\left\|\Phi^{k}\right\|_{L^{2}(0,1)}^{2}+\left|\Phi^{k}(0)\right|^{2}+\Delta t \int_{t_{k}}^{t_{k+1}}\left(\left\|y_{t t t t}(t)\right\|_{H^{1}(0,1)}^{2}\right.\right. \\
& \left.\left.+\left\|y_{t t t}(t)\right\|_{H^{1}(0,1)}^{2}+\left\|y_{t t}(t)\right\|_{H^{2}(0,1)}^{2}\right) d t\right]
\end{aligned}
$$

Additionally, using the Cauchy-Schwarz and Young inequalities, yields:

$$
\left|G^{k}\left(\Phi^{k+1}+\Phi^{k}\right)\right| \leq C\left(\left\|\Phi^{k+1}+\Phi^{k}\right\|_{L^{2}(0,1)}^{2}+\left|\Phi^{k+1}(0)+\Phi^{k}(0)\right|^{2}\right.
$$




$$
\left.+\frac{1}{\Delta t} \int_{t_{k}}^{t_{k+1}}\left(\left\|y_{t t}^{e}(t)\right\|_{L^{2}(0,1)}^{2}+\left|y_{t t}^{e}(0, t)\right|^{2}\right) d t+\left\|y_{t}^{e}\right\|_{C\left(\left[t_{k} ; t_{k+1}\right] ; H^{1}(0,1)\right)}^{2}\right) .
$$

Using the expressions (92)-(95), one obtains:

$$
\begin{array}{r}
\left\|U_{e}^{k+1}\right\|^{2}-\left\|U_{e}^{k}\right\|^{2} \leq C\left[\Delta t\left(\left\|U_{e}^{k+1}\right\|^{2}+\left\|U_{e}^{k}\right\|^{2}\right)\right. \\
+\Delta t\left\|y_{t}^{e}\right\|_{C\left(\left[t_{k} ; t_{k+1}\right] ; H^{1}(0,1)\right)}^{2}+\int_{t_{k}}^{t_{k+1}}\left(\left\|y_{t t}^{e}(t)\right\|_{L^{2}(0,1)}^{2}+\left|y_{t t}^{e}(0, t)\right|^{2}\right) d t \\
\left.+(\Delta t)^{3} \int_{t_{k}}^{t_{k+1}}\left(\left\|y_{t t t t}(t)\right\|_{H^{1}(0,1)}^{2}+\left\|y_{t t t}(t)\right\|_{H^{1}(0,1)}^{2}+\left\|y_{t t}(t)\right\|_{H^{2}(0,1)}^{2}\right) d t\right] .
\end{array}
$$

Let $m \in\{0,1, \ldots, s\}$. Suppose that $\Delta t \leq \frac{1}{2 C}$ (with $C$ from (96)). Summing (96) over $k \in\{0,1, \ldots, m\}$, gives:

$$
\begin{aligned}
\frac{1}{2}\left\|U_{e}^{m+1}\right\|^{2} \leq & \frac{3}{2}\left\|U_{e}^{0}\right\|^{2}+C\left(\Delta t \sum_{k=1}^{m}\left\|U_{e}^{k}\right\|^{2}+\left\|y_{t}^{e}\right\|_{C\left([0, T], H^{1}(0,1)\right)}^{2}\right. \\
& +\left\|y_{t t}^{e}\right\|_{L^{2}\left(0, T ; H^{1}(0,1)\right)}^{2}+(\Delta t)^{3}\left(\left\|y_{t t t t}\right\|_{L^{2}\left(0, T ; H^{1}(0,1)\right)}^{2}\right. \\
& \left.\left.+\left\|y_{t t t}\right\|_{L^{2}\left(0, T ; H^{1}(0,1)\right)}^{2}+\left\|y_{t t}\right\|_{L^{2}\left(0, T ; H^{2}(0,1)\right)}^{2}\right)\right) .
\end{aligned}
$$

Finally, due to the discrete in time Gronwall inequality and (84), we obtain:

$$
\begin{aligned}
\left\|U_{e}^{m+1}\right\|^{2} \leq & C\left(\left\|U_{e}^{0}\right\|^{2}+h^{2}\left(\left\|y_{t}\right\|_{C\left([0, T], H^{2}(0,1)\right)}^{2}+\left\|y_{t t}\right\|_{L^{2}\left(0, T ; H^{2}(0,1)\right)}^{2}\right)\right. \\
& +(\Delta t)^{3}\left(\left\|y_{t t t t}\right\|_{L^{2}\left(0, T ; H^{1}(0,1)\right)}^{2}+\left\|y_{t t t}\right\|_{L^{2}\left(0, T ; H^{1}(0,1)\right)}^{2}\right. \\
& \left.\left.+\left\|y_{t t}\right\|_{L^{2}\left(0, T ; H^{2}(0,1)\right)}^{2}\right)\right) .
\end{aligned}
$$

The result now follows from the previous expression, (82) and the triangle inequality.

\section{References}

[1] A. Pazy, Semigroups of Linear Operators and Applications to Partial Differential Equations, Springer-Verlag, New York (1983).

[2] R. Adams, Sobolev Spaces, Academic Press, New York (1975). 
[3] S.C. Brenner, L.R. Scott, The Mathematical Theory of Finite Element Methods, Springer Science + Business Media, New York (2008).

[4] H. Brezis, Analyse Fonctionnelle, Théorie et Applications, Masson, Paris (1983).

[5] P.G. Ciarlet, Introduction l'Analyse Numérique Matricielle et l'Optimisation, Masson, Paris (1982).

[6] G. Strang, G. Fix, An Analysis of the Finite Element Method, PrenticeHall, Englewood Cliffs, New Jersey (1973).

[7] G.H. Golub, C.F. Van Loan, Matrix Computations, The Johns Hopkins University Press, Baltimore (1989).

[8] J.L. Lions, E. Magenes, Nonhomogeneous Boundary Value Problems and Applications, Vol. 1, Springer, New York (1972).

[9] Z.H. Luo, B.Z. Guo, Ö. Mörgul, Stability and Stabilization of Infinite Dimensional Systems with Applications, Springer, London (1999).

[10] M.A. Naimark, Linear Differential Operators, Vol. 1, Springer, New York (1967).

[11] R. Temam, Infinite Dimensional Dynamical Systems in Mechanics and Physics, Vol. 68, Springer-Verlag, New York (1988).

[12] J. Rappaz, M. Picasso, Introduction l'Analyse Numérique, Presses Polytechniques et Universitaires Romandes, New York (1998).

[13] F.Z. Saouri, Stabilisation de Quelques Systèmes Élastiques. Analyse Spectrale et Comportement Asymptotique, Thèse de l'Université Henry Poincaré - Nancy I (2000).

[14] M. Miletic, Stability Analysis and a Dissipative FEM for an EulerBernoulli Beam with Tip Body and Passivity-Based Boundary Control, PhD Thesis, Vienna University of Technology (2015).

[15] A. Shkalikov, Boundary problem for ordinary differential operators with parameter in boundary conditions, J. Sov. Math., 33 (1986), 1311-1342.

[16] B. Chentouf, J.M. Wang, Optimal energy decay for a nonhomogeneous flexible beam with a tip mass, J. Dyn. Control Systems, 13, No 1 (2007), 37-53. 
[17] K.A. Touré, E.P. Mensah, M.M. Taha, Stabilization and Riesz basis property for an overhead crane model with feedback in velocity and rotating velocity, Journal of Nonlinear Analysis Application, 2014 (2014), 1-14.

[18] S.M. Choo, S.K. Chung, R. Kannan, Finite element Galerkin solutions for the strongly damped extensible beam equations, Korean. J. \& Appl. Math., 9, No 1 (2002), 27-43. 
